\title{
MAGNETIC ANOMALIES DUE TO 2-D CYLINDRICAL STRUCTURES - AN ARTIFICIAL NEURAL NETWORK BASED INVERSION
}

\author{
Bhagwan Das Mamidala $^{1}$ and Sundararajan Narasimman ${ }^{2}$ \\ ${ }^{1}$ Department of Mathematics, Osmania University, Hyderabad-500 007, India \\ ${ }^{2}$ Department of Earth Science, Sultan Qaboos University, Muscat, Oman
}

\begin{abstract}
Application of Artificial Neural Network Committee Machine (ANNCM) for the inversion of magnetic anomalies caused by a long-2D horizontal circular cylinder is presented. Although, the subsurface targets are of arbitrary shape, they are assumed to be regular geometrical shape for convenience of mathematical analysis. ANNCM inversion extract the parameters of the causative subsurface targets include depth to the centre of the cylinder $(Z)$, the inclination of magnetic vector $(\theta)$ and the constant term $(A)$ comprising the radius $(R)$ and the intensity of the magnetic field (I). The method of inversion is demonstrated over a theoretical model with and without random noise in order to study the effect of noise on the technique and then extended to real field data. It is noted that the method under discussion ensures fairly accurate results even in the presence of noise. ANNCM analysis of vertical magnetic anomaly near Karimnagar, Telangana, India, has shown satisfactory results in comparison with other inversion techniques that are in vogue.
\end{abstract}

\section{KEYWORDS}

Magnetic anomaly, Artificial Neural Network, Committee machine, Levenberg - Marquardt algorithm, Hilbert transform, modified Hilbert transform.

\section{INTRODUCTION}

In quantitative interpretation, the gravity and magnetic anomalies over a mineralized zone or geological structure can be approximated to simple geometrical shapes. Quantitative interpretation of the magnetic and gravity anomalies due to anticlines and synclines is accomplished by approximating them to two-dimensional, long horizontal circular cylinder. Linear concentrations of the mineral magnetite in a mineralized zone may be approximated some times to a horizontal cylinder. There are several methods of analyzing magnetic anomalies due to cylindrical structure. Parker Gay (1965) presented a set of master curves for the interpretation of the magnetic anomalies due to cylindrical bodies [24]. Rao et al. (1973) have developed direct methods for carrying out such interpretations [27]. Murthy and Mishra (1980) have proposed spectral approaches [22].

Mohan et al. (1990) used the Mellin transform in interpreting magnetic anomalies due to some two dimensional bodies [20]. Sundararajan et al. $(1985,1989)$ interpreted the magnetic anomalies of various components due to thin infinite dyke and spherical source by using Hilbert transform ([33], [34]). Srinivas (1998) used the modified Hilbert transform to interpret magnetic anomalies 
caused by 2-D horizontal circular cylindrical structures [31]. During 1999-2013, different methods Wavelet transform ([21], [15]), Displacement of the maximum and minimum by upward continuation [8], Euler deconvolution [11], Fraser filter [4], Hartley transform [17] and Direct analytic signal [5] were used for inversion of magnetic data.

TDX is a normalized version of the horizontal derivative filter and can recognize the edges of the shallow and deep bodies simultaneously. This filter is commonly used in the edge detection of potential field data. Recently, Alamdar et al. (2015) used combination of this balanced edge detection filter and Euler deconvolution to real magnetic data from Soork iron ore mine in Iran to estimate source location [1]. In the recent years, soft computing tools like Artificial neural network (ANN), Fuzzy logic, Genetic algorithm gained great importance in geophysical data inversion ([16], [32], [29], [13], [9], [25], [2]).

A committee machine consists of a group of intelligent systems named experts (ANN) and a combiner which combines the outputs of each expert [7]. Its advantages are more accuracy in prediction, speed learning and better generalization. If the combination of experts in committee machine were replaced by a single neural network, one would have a network with a correspondingly large number of adjustable weight parameters. The training time for such a large network is likely to be longer than for the case of a set of experts trained in parallel. Moreover, the risk of over fitting the data increases when the number of adjustable weight parameters is large compared to size of the set of the training data.

In this paper, the analysis of vertical magnetic anomalies due to a 2-D horizontal circular cylinder is carried out using ANN-based committee machine. The method is illustrated with the study of theoretical model and validity of procedure is tested with the addition of random noise to the source data. Further, the technique is exemplified with magnetic anomaly over a narrow band of quartz magnetic near Karimnagar, Telangana, India [31]. Both the theoretical as well as field data yield reasonably good results and are compared with other methods that are in vogue.

\section{Artificial NeUral Networks}

An artificial neural network consists of massively parallel interconnection of large number of neurons. It learns incrementally from environment to capture essential linear and nonlinear trends in complex data. On this basis it provides reliable predictions for new situations containing even noisy and partial information. ANN has at least two physical components, namely the processing elements and the connections between them. The processing elements are called neurons and connection between two neurons is called a link. Every link has a weight parameter associated with it. A neuron $(j)$ computes a single output $\left(a_{j}\right)$ from multiple inputs $\left(x_{1}, x_{2}, \ldots, x_{S_{0}}\right)$ by forming linear combination according to its input weights $\left(w_{j 1}, w_{j 2}, \ldots . ., w_{j s_{0}}, b_{j}\right)$ and then possibly putting the output through some activation function $(f()$.$) and is shown in Figure (1)$ ([19], [28], [7]), where $S_{0}$ is the number of inputs. Activation functions such as sigmoid are commonly used since they are nonlinear and continuously differentiable ([10], [30]).

Multi-layer perceptron (MLP) is a feed forward artificial neural network with one or more layers between input and output layers. Figure (2) shows a two-layer feed forward network. The net input to a neuron $j$ in layer $k+1$ is given by [6]:

$$
n_{j}^{k+1}=\sum_{i=1}^{S_{k}} w_{j i}^{k+1} a_{i}^{k}+b_{j}^{k+1}
$$


The output of neuron $j$ will be

$$
a_{j}^{k+1}=f_{j}^{k+1}\left(n_{j}^{k+1}\right) \quad \text { where } k=0,1,
$$

where $w_{j i}^{k+1}$ represents the weight associated with the $i$ 'th input to neuron $j$ in layer $k+1$, $b_{j}^{k+1}$ is a bias to neuron $j$ and $S_{k+1}$ is the number of neurons in the layer $k+1$. One may observe that if $\underline{x}=\left[\begin{array}{lllll}a_{1}^{0} & a_{2}^{0} & \ldots & \ldots & a_{S_{0}}^{0}\end{array}\right]^{T}$ is presented to the network and $A N(\underline{x})$ is the output of MLP, then

$$
A N(\underline{x})=\left[\begin{array}{llll}
a_{1}^{2} & a_{2}^{2} & \ldots & a_{S_{2}}^{2}
\end{array}\right]^{T}
$$

where $a_{j}^{2}$ 's are given by Eq. (2).

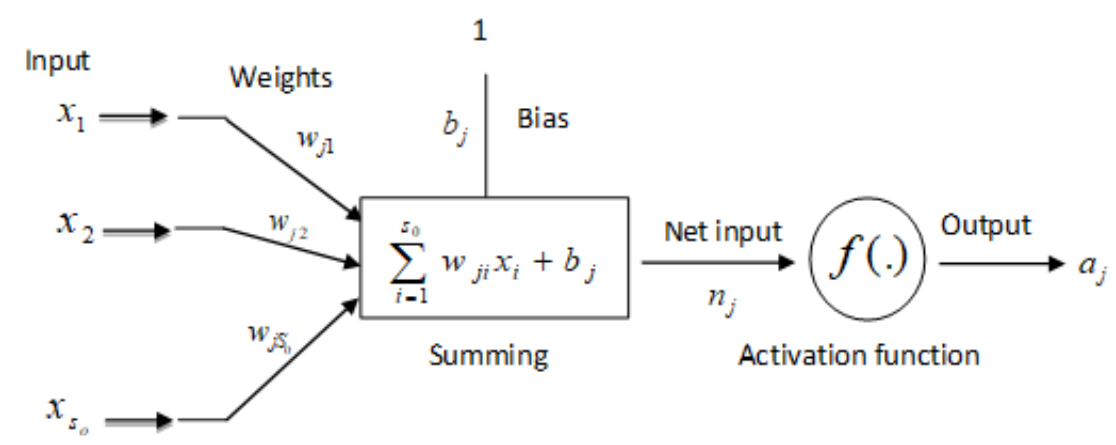

Figure 1 A model of an artificial neuron

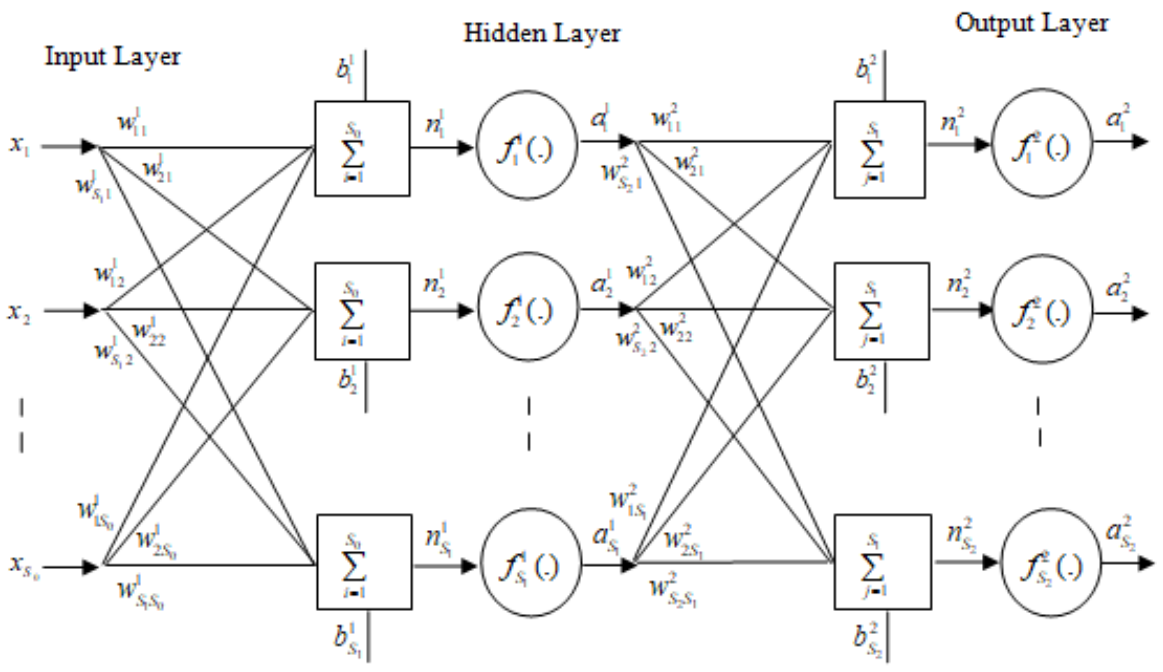

Figure 2 A two-Layer feed forward network (Multilayer perceptron)

MLP learns the problem behavior through a process called training and it would be taught with measured/simulated samples from a training set say $T=\left\{\left(\underline{x}_{1}, \underline{t}_{1}\right),\left(\underline{x}_{2}, \underline{t}_{2}\right), \ldots,\left(\underline{x}_{P}, \underline{t}_{P}\right)\right\}$. The performance (Perf) of MLP is calculated using the following error function: 


$$
E(\vec{w})=\frac{1}{2} \sum_{p=1}^{P}\left(t_{p}-A N\left(\underline{x}_{p}\right)\right)^{T}\left(t_{p}-A N\left(\underline{x}_{p}\right)\right)=\frac{1}{2} \sum_{p=1}^{P} e_{p}^{T} e_{p}
$$

where $A N\left(\underline{x}_{p}\right)$ is the output of network, $\vec{w}$ is the weight vector containing all the weights of the network, $t_{p}$ - target, $e_{p}$ - error and $P$ - total number of training samples. The goal of the training is to find the weights that will impact the output from MLP to match the targets as closely as possible. If the outputs of MLP come as close as possible to match the targets for all the samples, then performance function $E(w)$ of network is minimized. Levenberg-Marquardt back-propagation algorithm ([14], [18], [6]) is one of the numerical optimization techniques that minimizes $E(w)$. It is fast with stable convergence. Levenberg-Marquardt algorithm (LMA) [3] is given by:

$$
\vec{w}(n+1)=\vec{w}(n)-\left(J(\vec{w})^{T}(n) J(\vec{w})(n)+\mu I\right)^{-1} J(\vec{w})^{T}(n) e(\vec{w})(n)
$$

where $e(\vec{w})=\left[\begin{array}{llllll}e_{1}(\vec{w}) & e_{2}(\vec{w}) & \ldots & \ldots & e_{P}(\vec{w})\end{array}\right]^{T}$ is the error vector comprising the errors for all the training samples, $J(\vec{w})(n)$ is a Jacobian matrix, $n$ is an iteration number and $\mu$ is a damping parameter. When $\mu$ is large, the method takes a small step in the gradient direction. As the method nears a solution, $\mu$ is chosen to be small and the method converges quickly via the Gauss Newton method. The flowchart of implementation of the LM algorithm is shown in Figure (3).

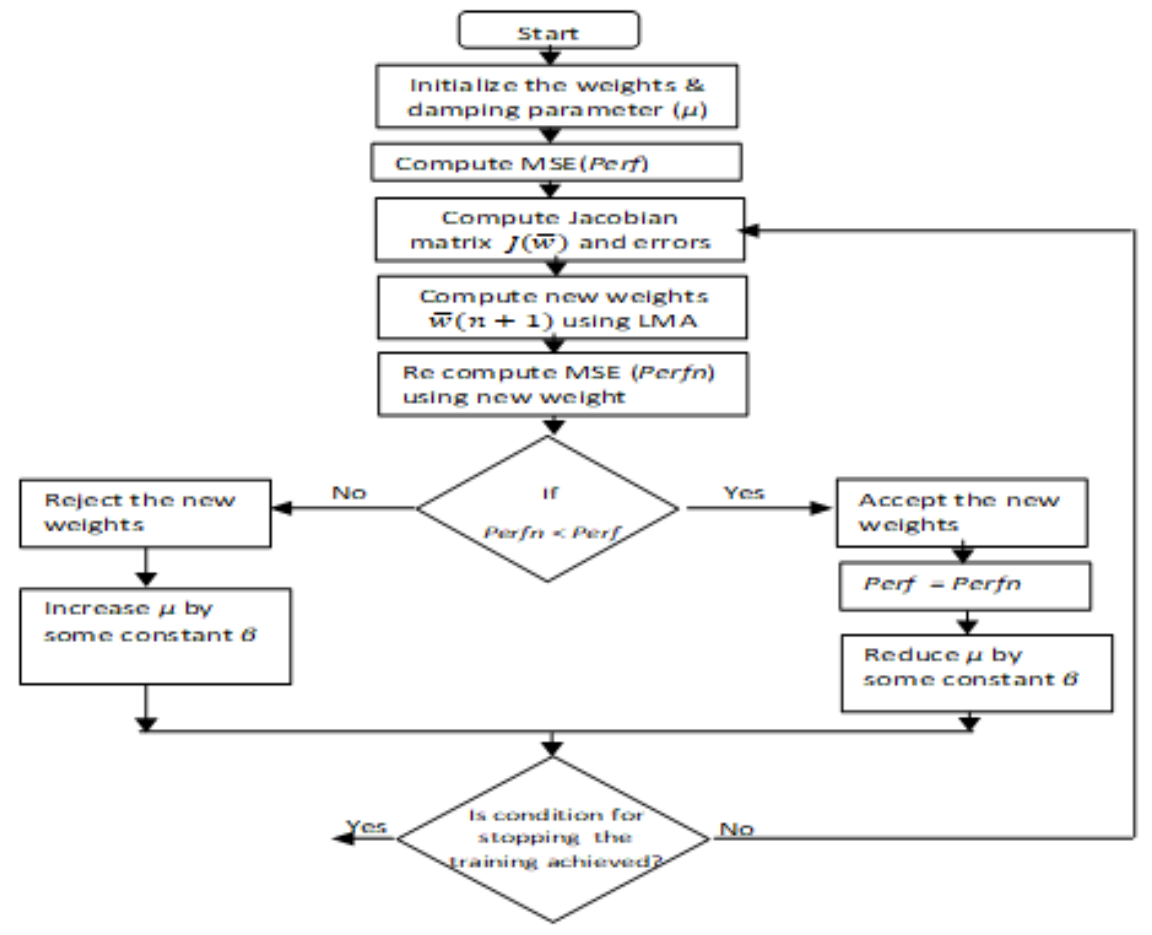

Figure 3 Flow-chart of implementation of the LM algorithm

A method for calculation of $\vec{w}(n+1)$ using Eq. (5) requires both forward and backward calculations. First, feed forward calculations which are made to determine the error at the output layer. The elements of the Jacobian matrix are then obtained by propagating this error back through the network which can be computed by a simple modification to the standard back 
propagation algorithm [6]. The back propagation process has to be repeated for every output separately in order to obtain consecutive rows of the Jacobian matrix.

\subsection{ARtificial Neural Network Committee Machine (ANNCM)}

Committee machines with static structure, the outputs of several predictors (expert) are combined by a mechanism that does not involve the input signal with ensemble and boosting methods. Figure (4) shows a number of differently trained neural networks (i.e., experts), which share a common input and whose individual outputs are combined using rules such as averaging, voting etc., to produce an overall output. Such a technique is referred to as an ensemble averaging method. This method is most popular [23]. Ensemble averaging creates a group of networks (experts); each with low bias and high variance, then combines them to a new network with low bias and low variance. Further the idea behind such network is to fuse knowledge acquired by experts in order to arrive at an overall decision that is superior to that of any of the individual experts ([12], [23], [7]).

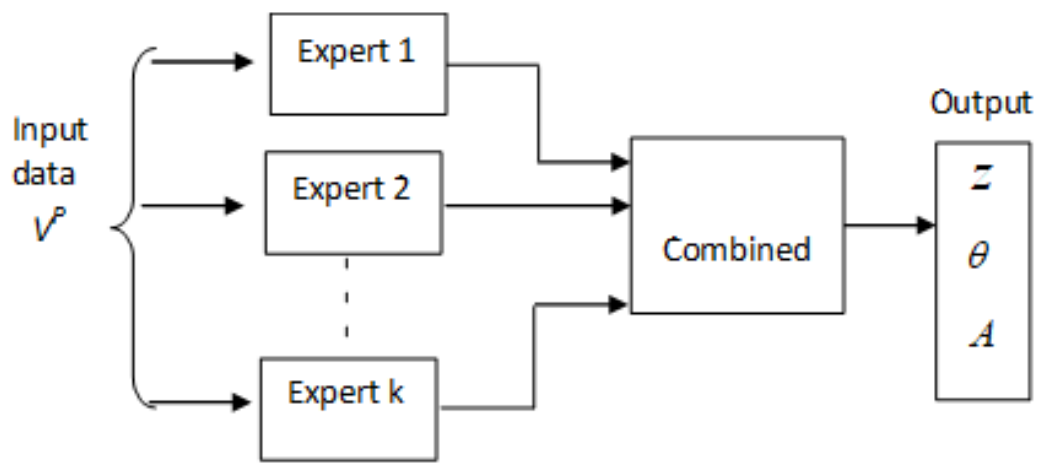

Figure 4 ANN Committee machine

\section{Magnetic Effect Due To A 2-D Horizontal Circular Cylinder}

The vertical magnetic effect $V(x)$ due to a 2-D horizontal circular cylinder extending infinitely along the $\mathrm{Y}$-axis with its normal section parallel to the $\mathrm{X}-\mathrm{Z}$ plane (Figure (5)) at a point ' $x$ ' is given by [36]:

$$
V(x)=A\left[\frac{\left(z^{2}-x^{2}\right) \sin \theta-2 x z \cos \theta}{\left(x^{2}+z^{2}\right)^{2}}\right]
$$

where,

$\mathrm{z}$ - is the depth to the centre of the cylinder,

$\theta$ - is the inclination of magnetic vector,

$A$-is the constant term comprising the radius $(R)$ and the intensity of the magnetic field

(I) and is given as $A=2 \pi R^{2} I$. 


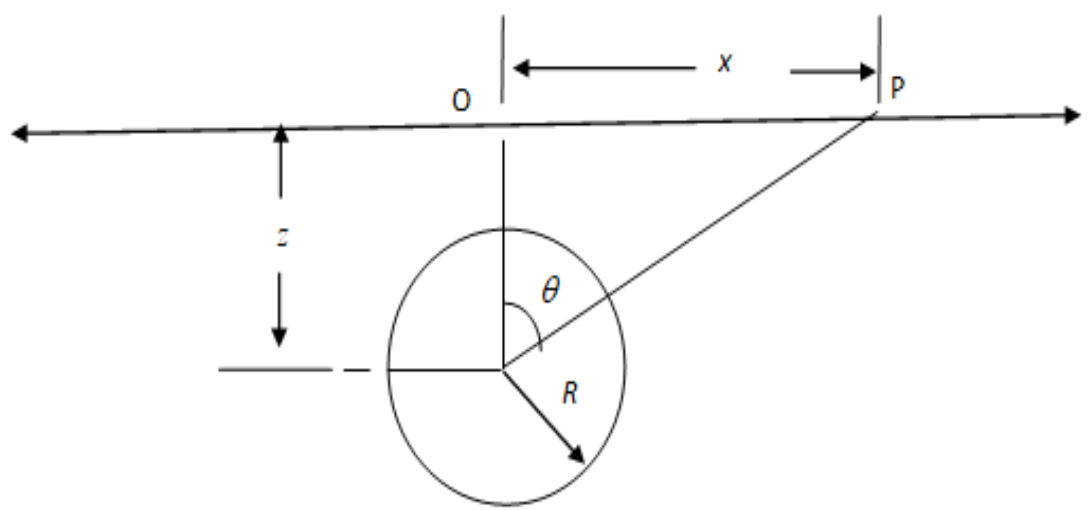

Figure 5 Geometry of the 2D Horizontal Circular Cylinder

The inversion of magnetic effect due to a 2-D horizontal circular cylinder is achieved by ANNCM which consists of phase-I and phase-II and is discussed in the following subsections. In phase-I coarse values of parameters are obtained whereas in phase-II fine values of parameters are obtained. We call, phase-I and phase-II as coarse and fine application. Flow chart of phase-I and phase-II is shown in Figure (6).

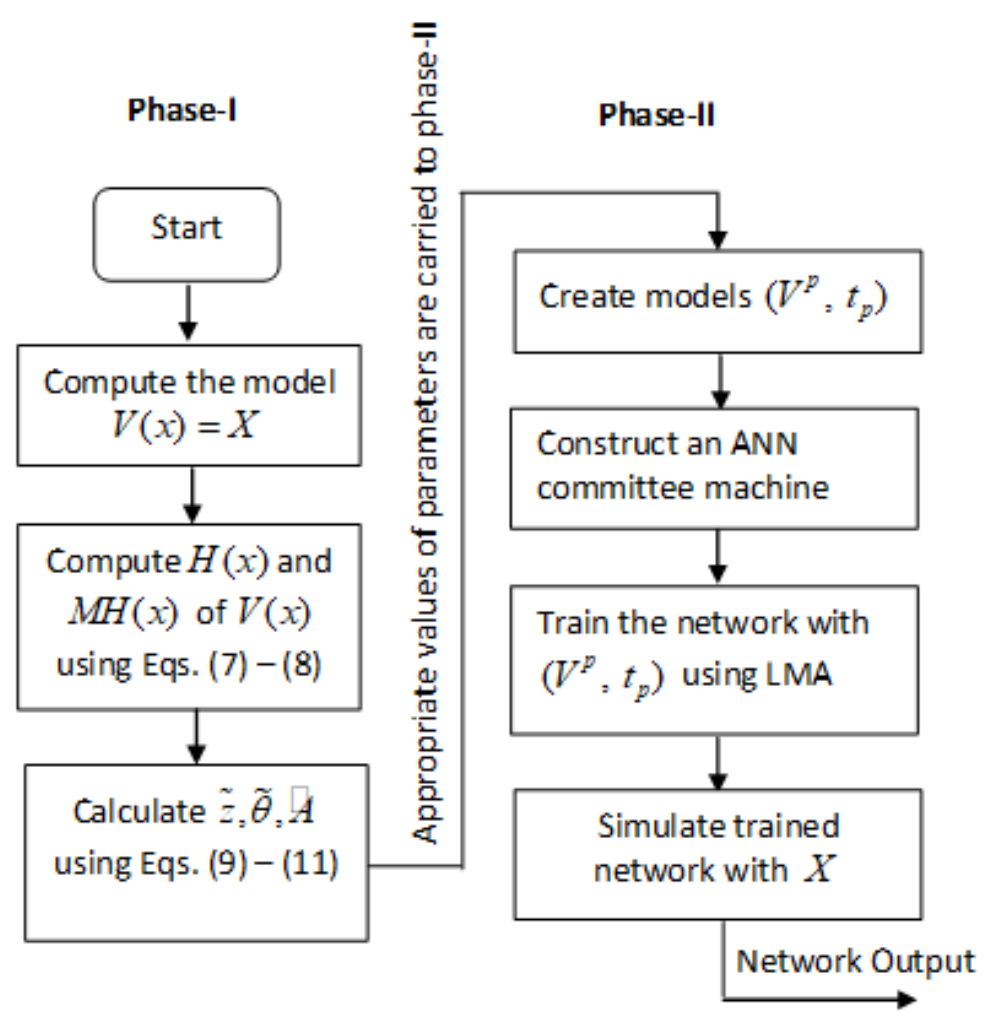

Figure 6 Flow-chart of implementation of the Phase-I and Phase-II 


\subsection{Phase-I}

In phase-I, Hilbert and modified Hilbert transform are used for the analysis of vertical magnetic anomaly $(V(x)=X)$ generated with the model parameters $\left(z_{o}, \theta_{o}, A_{o}\right)$. The Hilbert transform $H(x)$ and the modified Hilbert transform $M H(x)$ of the vertical magnetic anomaly $V(x)$ due to an inclined sheet are computed by [31]:

$$
\begin{aligned}
& H(x)=-A\left[\frac{\left(z^{2}-x^{2}\right) \cos \theta+2 x z \sin \theta}{\left(x^{2}+z^{2}\right)^{2}}\right] \\
& M H(x)=-A\left[\frac{\left(z^{2}-x^{2}\right) \cos \theta-2 x z \sin \theta}{\left(x^{2}+z^{2}\right)^{2}}\right]
\end{aligned}
$$

Sundararajan and Srinivas [35] reported in literature that the Hilbert transform and its modified version intersect exactly over the origin (centre of the subsurface target). From the equations of vertical magnetic anomaly $V(x)$ and the modified Hilbert transform $M H(x)$, the depth to the top of the sheet $(\tilde{z})$, the inclination $(\tilde{\theta})$ and the constant term $(\widetilde{A})$ are given as:

$$
\begin{gathered}
\tilde{z}=-\frac{x_{1}+x_{2}}{2} \\
\tilde{\theta}=\tan ^{-1}\left[\frac{2 z x M H(x)-\left(z^{2}-x^{2}\right) V(x)}{\left(z^{2}-x^{2}\right) M H(x)-2 z x V(x)}\right] \\
\tilde{A}=z^{2} \sqrt{V(0)^{2}+M H(0)^{2}}
\end{gathered}
$$

where $x_{1}$ and $x_{2}$ are the abscissa of the points of intersection of $V(x)$ and $M H(x)$.

The appropriate values $\tilde{z}, \tilde{\theta}, \widetilde{A}$ of parameters obtained by equations (9) - (11) will be carried to phase-II in order to increase their accuracy.

\subsection{Phase-II}

Phase-II can be implemented in stepwise as hereunder.

Step -1: Create models to train an ANN in the following way.

Let $Z=\left\{\tilde{z}_{1}, \tilde{z}_{2}, \ldots, \tilde{z}_{m_{1}}\right\}, \quad I=\left\{\tilde{\theta}_{1}, \tilde{\theta}_{2}, \ldots, \tilde{\theta}_{m_{2}}\right\}$ and $C=\left\{\widetilde{A}_{1}, \tilde{A}_{2}, \ldots, \tilde{A}_{m_{3}}\right\}$, and be three sets of parameters that are selected in a small neighborhood of $\widetilde{Z}$. $\widetilde{\boldsymbol{\theta}}$ and $\widetilde{\boldsymbol{A}}$ respectively. Then the number of models of type $\left(\tilde{\boldsymbol{z}}_{i}, \tilde{\boldsymbol{\theta}}_{j}, \widetilde{\boldsymbol{A}}_{k}\right)$ is $m_{1} \times m_{2} \times m_{3}$, where $\tilde{z}_{i} \in Z, \quad \tilde{\theta}_{j} \in I$ and $\tilde{A}_{k} \in C$.

For simplicity, rename each $\left(\tilde{\boldsymbol{z}}_{i}, \tilde{\boldsymbol{\theta}}_{j}, \widetilde{A}_{k}\right)$ as $\left(z_{p}, \theta_{p}, A_{p}\right)$ for $p=1,2, \ldots ., P$, where $P=m_{1} \times m_{2} \times m_{3}$. Let $V^{p}\left(x_{j}\right)$ be vertical magnetic effect due to a 2-D horizontal circular 
cylinder generated by Eq. (6) at a point $x_{j}$ with the model parameters $\left(z_{p}, \theta_{p}, A_{p}\right)$ where $1 \leq p \leq P, 1 \leq j \leq S_{0}$. Let $V^{p}=\left(V^{p}\left(x_{1}\right), \ldots, V^{p}\left(x_{j}\right), \ldots, V^{p}\left(x_{S_{0}}\right)\right), t_{p}=\left(z_{p}, \theta_{p}, A_{p}\right)$ and

$$
T=\left\{\left(V^{1}, t_{1}\right),\left(V^{2}, t_{2}\right), \ldots,\left(V^{P}, t_{P}\right)\right\}
$$

Realization of an inversion estimation of parameters of the anomaly $(X)$ is achieved by training an ANNCM with the models $\left(V^{p}, t_{p}\right)$, where $\left(V^{p}, t_{p}\right) \in T,(p=1,2, \ldots . ., P)$.

Step-2: In this step, first design an artificial neural network committee machine with suitable number of experts (MLPs) and in turn each will be trained in batch mode with the models $\left(V^{p}, t_{p}\right),(p=1,2, \ldots . ., P)$ using LM algorithm.

\section{THEORETICAL MODELS}

The vertical magnetic anomaly $V(x)$ due to a $2 \mathrm{D}$ horizontal circular cylinder of theoretical model-I is generated using Eq. (6) with input parameters $(z=12, \theta=40$ and $A=800)$ consisting of 51 samples with 2 units as sampling. The appropriate values of parameters obtained in phase-I are: $\tilde{z}=8.96, \tilde{\theta}=36.24^{\circ}$ and $\widetilde{A}=485.26$. The range of parameters and number of steps that were used in phase-II to generate ANN models $\left(V^{p}, t_{p}\right)$ are given in Table (1). The ANNCM with five MLPs (Figure (4)) of same topology (i.e., number layers, number of neurons in each layer are same) with different initial weights is used to invert the model-I by assigning 51 samples $V^{p}=\left[\begin{array}{llllll}V^{p}\left(x_{1}\right) & \ldots & \ldots & \ldots & V^{p}\left(x_{51}\right)\end{array}\right]^{T}$ to the input layer. Ten neurons with hyperbolic tangent transfer functions are used for hidden layer. Three neurons with linear transfer functions are used for output layer to extract the required parameters $(z, \theta, A)$. While training the networks the set $T=\left\{\left(V^{1}, t_{1}\right),\left(V^{2}, t_{2}\right), \ldots,\left(V^{P}, t_{P}\right)\right\}$ is randomly divided into three subsets namely training, validation and testing sets, each are containing $70 \%, 15 \%$ and $15 \%$ models respectively. The performance of each MLP is calculated using Eq. (4) and weights are adjusted according to Eq. (5). Output of ANNCM is computed by ensemble averaging method and given in Table (2). The vertical magnetic anomaly $V(x)$ and the ANNCM inversion response are shown in Figure (7). The Hilbert transform $H(x)$ and the modified Hilbert transform $M H(x)$ of the vertical magnetic anomaly $V(x)$ are computed and shown in Figure (8). The well trained network can invert any data that falls within the training range in almost no time.

Theoretical Examples MODEL-I

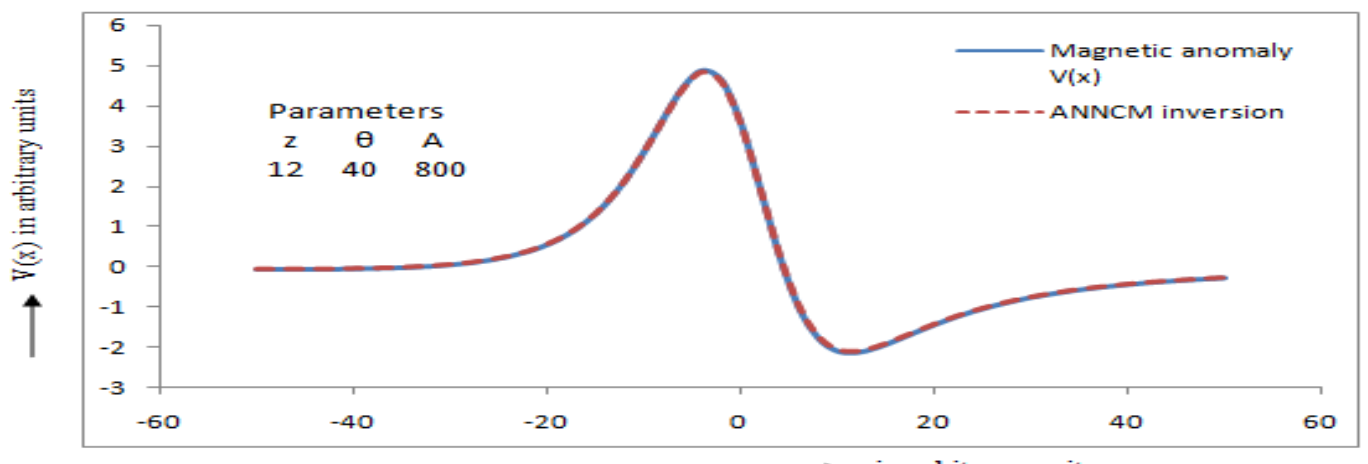

Figure 7 Vertical magnetic anomaly $\mathrm{V}(\mathrm{x})$ and ANNCM inversion response of model-I 


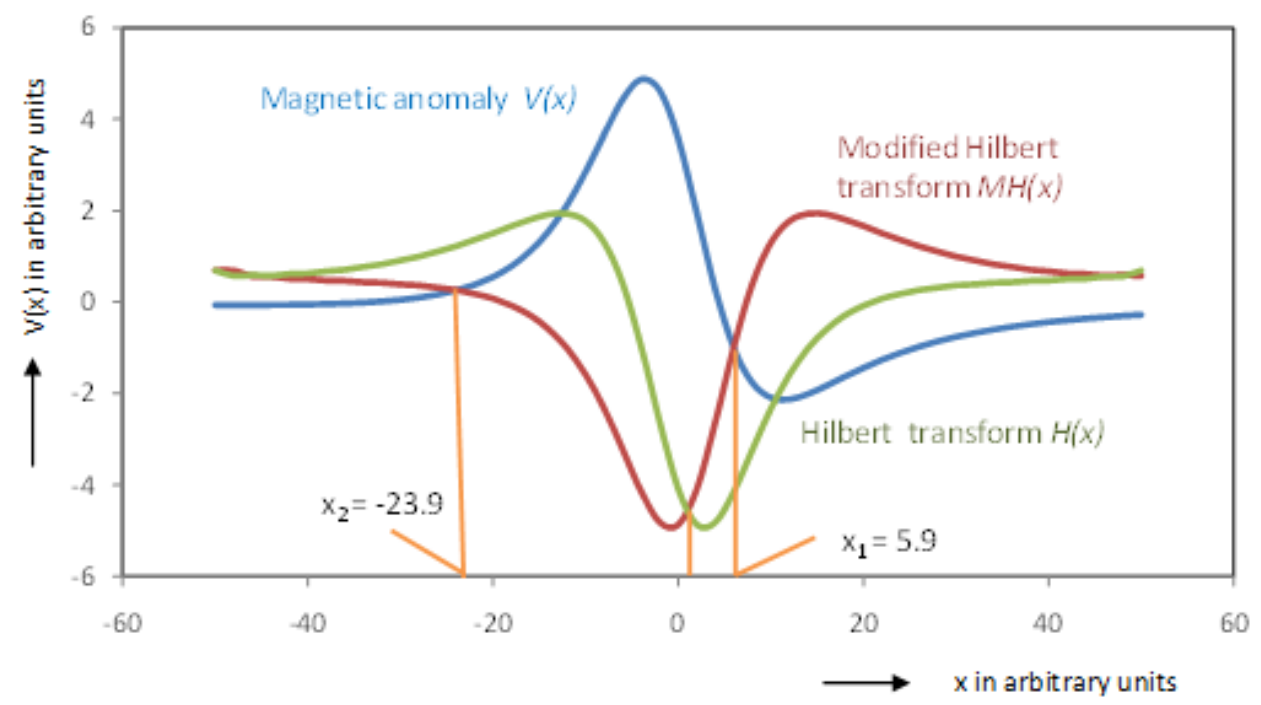

Figure 8 Vertical magnetic anomaly the Hilbert transform and modified Hilbert transform of model-I

\subsection{EFFECT OF RANDOM NOISE}

Ten percent of Gaussian random noise is added to the vertical magnetic anomaly $V(x)$ of modelI [Figure (7)] and is shown in Figure (9). As in the case of noise free analysis, of magnetic anomalies, the values of parameters obtained during phase-I are $\tilde{z}=9.61, \widetilde{\boldsymbol{\theta}}=36.47^{\circ}$ and $\widetilde{A}=\mathbf{4 2 4 . 5 1}$. The range of parameters, number of steps and the number of ANN models that are generated in phase-II are given in Table (1). The ANNCM inversion response is shown in Figure (9). The Hilbert transform $H(x)$ and the modified Hilbert transform $M H(x)$ of the noisy vertical magnetic anomaly $V(x)$ are computed and shown in Figure (10). The result of the ANNCM inversion parameters is given in Table (2).

Theoretical Example with noise MODEL-II

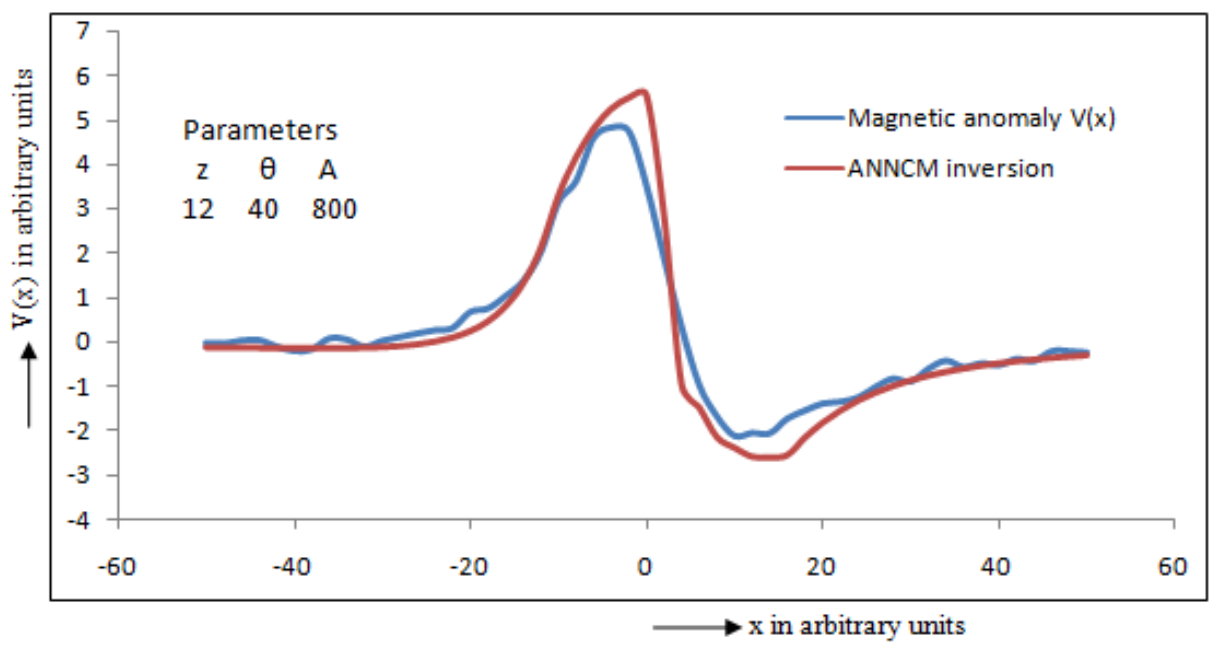

Figure 9 Noisy vertical magnetic anomaly and ANNCM inversion response of model-II 


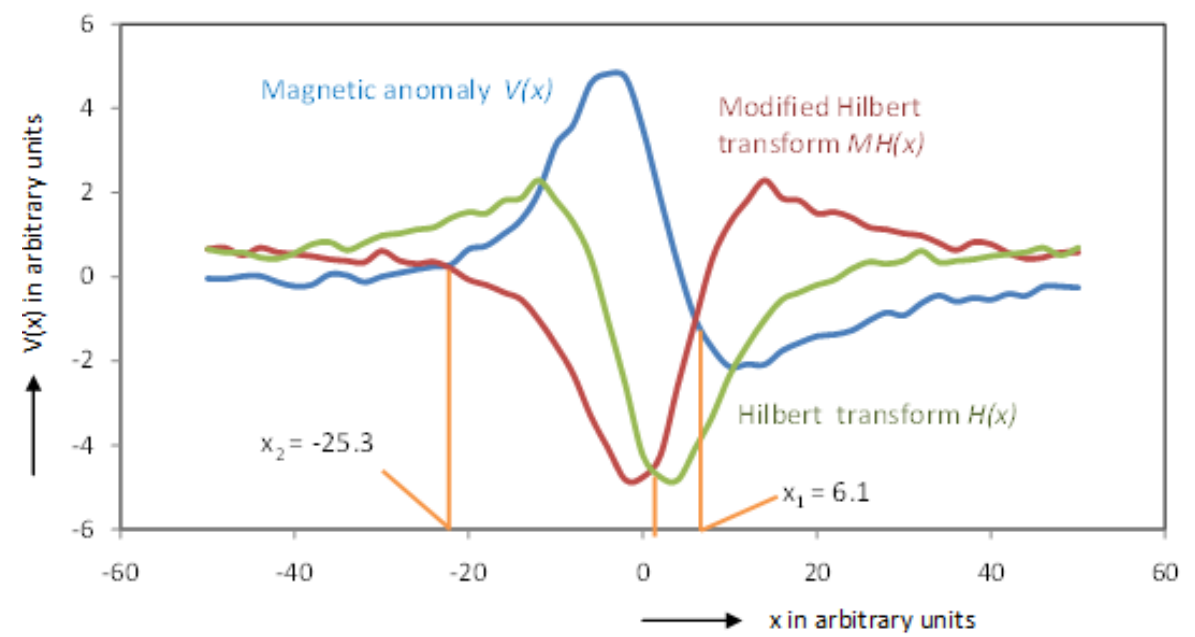

Figure 10 Vertical magnetic anomaly $\mathrm{V}(\mathrm{x})$ the Hilbert transform $\mathrm{H}(\mathrm{x})$ and modified Hilbert transform MH(x) of model-II.

Table 1 Range of input parameters and number of ANN generated models (Phase-II)

\begin{tabular}{|c|c|c|c|c|}
\hline Examples & $z$ & $A$ & $\begin{array}{l}\text { Number } \\
\text { of ANN } \\
\text { models }\end{array}$ \\
\hline Model-I & $6-12$ & $31^{\circ}-41^{\circ}$ & $405-565$ & $\mathbf{2 7 0}$ \\
& $(5)$ & $(6)$ & $(9)$ & \\
\hline $\begin{array}{c}\text { Model-II } \\
\text { (with noise) }\end{array}$ & $6.6-12.6$ & $31.4^{\circ}-41.4^{\circ}$ & $374-474$ & $\mathbf{3 0 0}$ \\
$(5)$ & $(6)$ & $(10)$ & \\
\hline
\end{tabular}

\begin{tabular}{|c|c|c|c|c|}
\hline & Parameters & $\mathrm{z}^{*}$ & $\theta$ & $\mathrm{A}^{*}$ \\
\hline \multirow{3}{*}{$\begin{array}{c}\text { Theoretical } \\
\text { Model-I }\end{array}$} & Assumed values & 12.00 & $40^{\circ} 00^{\prime}$ & 800.00 \\
\hline & $\begin{array}{l}\text { ANNCM processed } \\
\text { parameters }\end{array}$ & 12.05 & $40^{\circ} 02^{\prime}$ & 799.60 \\
\hline & $\begin{array}{l}\text { Error in } \\
\text { percentage }\end{array}$ & 0.41 & 0.08 & 0.05 \\
\hline \multirow{3}{*}{$\begin{array}{c}\text { Theoretical } \\
\text { Model with } \\
\text { Noise }\end{array}$} & Assumed Values & 12.00 & $40^{\circ} 00^{\prime}$ & 800.00 \\
\hline & $\begin{array}{l}\text { ANNCM processed } \\
\text { parameters }\end{array}$ & 10.85 & $41^{\circ} 17^{\prime}$ & 872.93 \\
\hline & $\begin{array}{l}\text { Error in } \\
\text { percentage }\end{array}$ & 9.58 & 3.20 & 9.11 \\
\hline
\end{tabular}

Table 2 Theoretical examples (* in arbitrary units)

\section{FieLd EXAMPLe}

The applicability of the proposed technique is demonstrated on an observed vertical magnetic data near Karimnagar district, Telangana, India (Srinivas 1998) and is shown in Figure (11). The 
total length of the profile (182.60 meters) was digitized into 60 equal parts at an interval of $3.0433 \mathrm{~m}$. The quality of the data is determined by the signal to noise ratio $(S N R)$ and is given as:

$$
S N R=\frac{m}{s},
$$

where $m$ is the mean and $s$ is the standard deviation of the data. If the ratio is less than 3 , the data is assumed to be very poor quality. If the ratio is greater than 3, then the level of noise is negligible and the data shall be considered clean. Signal to noise ratio of the field data is calculated and is given by:

$$
S N R=\frac{5017.1}{646.8136}=7.7567
$$

The appropriate values of parameters obtained in phase-I are $\tilde{z}=24.38 \mathrm{~m}, \tilde{\theta}=73^{\circ}$, $\widetilde{A}=46592000$ Three hundred training models were created by assigning different values to $(z, \theta, A)$ in a close range of $(\tilde{z}, \widetilde{\theta}, \widetilde{A})$ which were used in phase-II are as follows:

- the depth $z(19 m-29 m)$, with five points in this range

- the inclination $\theta\left(70^{\circ}-80^{\circ}\right)$, with six points in this range

- the constant $A$ (46562000 - 46622000), with ten points in this range;

ANNCM inversion response compared with the field data are shown in Figure (11). The Hilbert transform $H(x)$ and the modified Hilbert transform $M H(x)$ of the vertical magnetic anomaly $V(x)$ are computed and shown in Figure (12). The estimated parameters are given in Table (3). Results shown are better and agree well with other inversion methods (Table 3).

\begin{tabular}{|c|c|c|c|}
\hline Methods & $\mathbf{z}$ (in meters) & $\theta$ & $A$ \\
\hline Gradient method [26] & 23.23 & $56^{\circ} 00^{\prime}$ & $\ldots$ \\
\hline $\begin{array}{c}\text { Modified Hilbert } \\
\text { transform Technique } \\
{[31]}\end{array}$ & 21.4 & $46^{\circ} 00^{\prime}$ & $\ldots$. \\
\hline $\begin{array}{c}\text { Present Artificial Neural } \\
\text { Network Committee } \\
\text { Machine }\end{array}$ & 22.66 & $78^{\circ} 18^{\prime}$ & 47493187.04 \\
\hline
\end{tabular}

Table 3 Field Example (Vertical magnetic anomaly, near Karimnagar, Telangana, India)

\section{RESUlTS AND DISCUSSION}

During training network, 670 training models were used for both theoretical and field data for which Levenberg-Marquart algorithm is very much suitable. From Table (2), it is observed that the results in general agree with the assumed values. However, the addition of random noise level to the magnetic anomaly and subsequent analysis show a marginal variation implying that the effect of such noise is almost negligible in the present method. The method with LM algorithm shows the best performance in extraction of parameters of a model. Hence, determination of the depth and inclination of various structures from magnetic data can be solved effectively. 


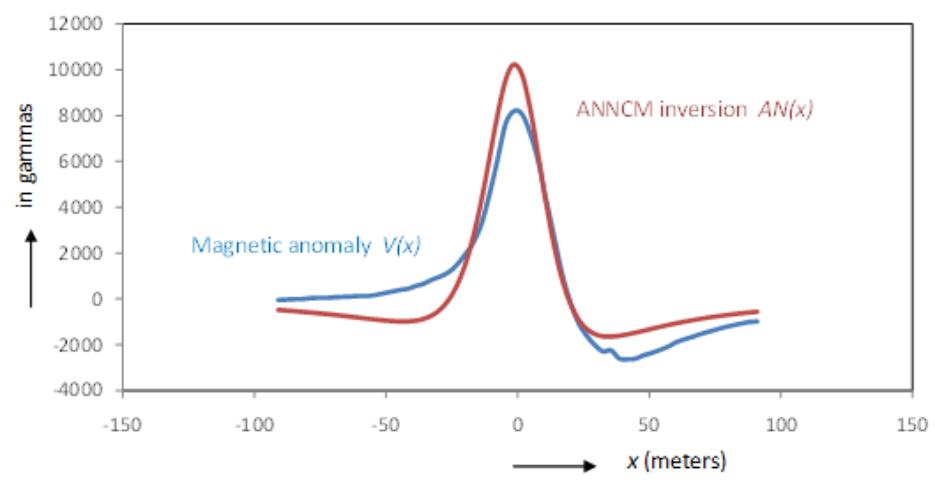

Figure 11 Field Example Vertical magnetic anomaly over a narrow band of quartz magnetite, near Karimnagar District, Telangana, India.

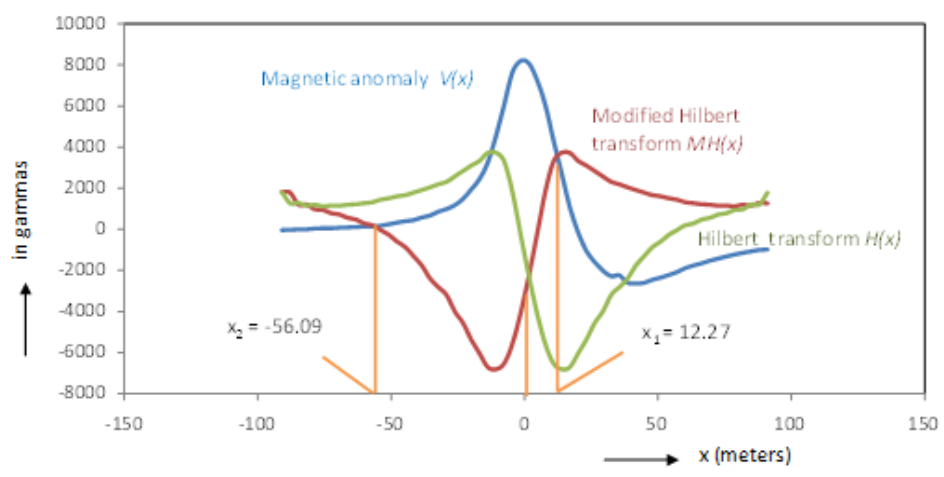

Figure 12 Vertical magnetic anomaly the Hilbert transform and modified Hilbert transform of field data.

\section{Conclusions}

The accuracy of ANNCM inversion results is fairly good. The ANN committee machine analysis of magnetic inversion is simple and elegant and the method is effective even in the presence of noise. In addition, it is independent of analytical nature of the data.

\section{REFERENCES}

[1] Alamdar K, Kamkare-Rouhani A \& Ansari A H, (2015) "Interpretation of the magnetic data from anomaly $2 \mathrm{c}$ of Soork iron ore using the combination of the Euler deconvolution and TDX filter", Arabian Journal of Geosciences, Vol. 8, No.8, pp 6021-6035.

[2] Amjadi A \& Naji J, (2013) "Application of genetic algorithm optimization and least square method for depth determination from residual gravity anomalies", International Research Journal of Applied and Basic Sciences, Science explorer publications, Vol. 5, No.5, pp 661- 666.

[3] Edwin K P, Chong \& Stanislaw H Zak,(2001) An introduction to optimization, Second edition, A Willey-Interscience Publication.

[4] Fitian R Al-Rawi, (2009) "Magnetic depth estimation of dyke- like bodies by using Fraser filter", Journal of Al-Anbar University for Pure Science, Vol. 3, No.1, pp 89-97.

[5] Guoqing Ma \& Lili Li., (2013) "Direct analytic signal (DAS) method in the interpretation of magnetic data", Journal of Applied Geophysics, Vol. 88, pp 101-104.

[6] Hagan M T \& Menhaj M B., (1994) "Training feedforward networks with the Marquardt algorithm", IEEE Transactions on Neural Networks, Vol. 5, No.6, pp 989-993.

[7] Haykin S., (1999) Neural networks, A Comprehensive Foundation, 2nd edition, Prentice Hall, New Jersey, 842pages. 
[8] Ibrahim Kara, Mustafa Ozdemir \& Ali Ismet Kanli, (2003) "Magnetic interpretation of horizontal cylinders using displacement of the maximum and minimum by Upward Continuation", Journal of the Balkan Geophysical Society, Vol. 6, No.1, pp 16-20.

[9] Kaftan I, Sindirgi P \& Akdemir O, (2014) "Inversion of self potential anomalies with multilayer perceptron neural networks", Pure and Applied Geophysics, Vol.171, No.8, pp 1939-1949.

[10] Karlik B \& Olgac A V, (2011) "A performance analysis of various activation functions in generalized MLP architectures of neural networks", International Journal of Artificial Intelligence and Expert Systems, Vol.1, No.4, pp 111-122.

[11] Keating P \& Pilkington M, (2004) "Euler deconvolution of the analytic signal and its application to magnetic interpretation", Geophysical Prospecting, Vol.52, pp 165-182.

[12] Krasnopolsky V M, (2007) "Reducing uncertainties in neural network Jacobians and improving accuracy of neural network emulations with ensemble approaches", Neural Networks, Vol.20, No.4, pp 454-461.

[13] Lashin A \& Din S S El, (2013) "Reservoir parameters determination using artificial neural networks: Ras Fanar field, Gulf of Suez, Egypt”, Arabian Journal of Geosciences, Vol.6, No.8, pp 2789-2806.

[14] Levenberg K, (1944) "A method for the solution of certain nonlinear problems in least squares", Quarterly of Applied Mathematics, Vol.2, pp 164-168.

[15] Li Y \& Oldenburg D W, (2003) "Fast inversion of large scale magnetic data using wavelet transforms and a logarithmic barrier method", Geophysical Journal International, Vol.152, pp 251-265.

[16] Mansour A Al-Garni, (2009) "Interpretation of some magnetic bodies using neural networks inversion”, Arabian Journal of Geosciences, Vol.2, No.2, pp 175-184.

[17] Mansour A Al-Garni, (2011) "Spectral analysis of magnetic anomalies due to a 2-D horizontal circular cylinder, A Hartley transforms technique", SQU Journal for Science, Vol.16, pp 45-56.

[18] Marquardt D, (1963) "An algorithm for least-squares estimation of nonlinear parameters", Journal of the Society for Industrial and Applied Mathematics, Vol.11, No.2, pp 431-441.

[19] McCulloch W S \& Pitts W, (1943) "A logical calculus for ideas imminent in nervous activity", Bulletin of Mathematical Biophysics, Vol. 5, pp 115-133.

[20] Mohan N L, Babu L, Sundararajan N \& Seshagiri Rao S V, (1990) "Analysis of magnetic anomalies due to some two dimensional bodies using the Mellin transform", Pure and Applied Geophysics, Vol.133, pp 403-428.

[21] Moreau F, Gilbert D, Holschneider M \& Saracco G, (1999) "Identification of sources of potential fields with continuous wavelet transform, Basic theory”, J. Geophys. Res, Vol.104, pp 5003-5013.

[22] Murthy K S R \& Mishra D C, (1980) "Fourier transform of the general expression for the magnetic anomaly due to long horizontal cylinder", Geophysics, Vol.45, pp 1091-1093.

[23] Naftaly U, Intrator N \& Horn D, (1997) "Optimal ensemble averaging of neural networks", Network: Computation in Neural Systems, Vol.8, pp 283-296.

[24] Parker Gay Jr. S, (1965) "Standard curves for magnetic anomalies over long horizontal cylinders", Geophysics, Vol.30, pp 818-828.

[25] Pourghasemi H R, Pradhan B \& Gokceoglu C, (2012) "Application of fuzzy logic and analytical hierarchy process (AHP) to landslide susceptibility mapping at Haraz watershed, Iran", Natural Hazards., Vol.63, No.2, pp 965-996.

[26] Radhakrishna Murthy I V, Visweswara Rao C \& Gopala Krishna C, (1980) "A gradient method for interpreting magnetic anomalies due to horizontal circular cylinders, infinite dykes and vertical steps", Journal of Earth System Science, Vol. 89, No. 1, pp 31-42.

[27] Rao B S R, RadhaKrishna Murthy I V \& Visweswara Rao, (1973) “A direct method of interpreting gravity and magnetic anomalies, The case of a horizontal cylinder”, PAGEOPH, Vol.102, pp 67-72.

[28] Rosenblatt F, (1958) "The perceptron: A probabilistic model for information storage and organization in the brain", Psychological Review, Vol. 65, pp 386-408.

[29] Saumen Maiti, Vinit C Erram, Gautam Gupta \& Ram Krishna Tiwari, (2012) "ANN based inversion of DC resistivity data for groundwater exploration in hard rock terrain of western Maharashtra (India)". Journal of Hydrology, Vols.464-465: pp 294-308.

[30] Sibi P, Allwyn Jones S \& Siddarth P, (2013) "Analysis of different activation functions using back propagation neural networks", Journal of Theoretical and Applied Information Technology, Vol.47, No.3, pp 1264-1268.

[31] Srinivas Y., (1998) Modified Hilbert transform- A tool to the interpretation of geopotential field anomalies, Thesis, Osmania University, Hyderabad, India. 
[32] Srinivas Y, Stanley Raj A, Muthuraj D, Hudson Oliver D \& Chandrasekar N, (2010) “An application of artificial neural network for the interpretation of three layer electrical resistivity data using feed forward back propagation algorithm", Current Development in Artificial Intelligence, Vol.1, No.1-3, pp 1-11.

[33] Sundararajan N, Mohan N L, Vijaya Raghava M S \& Seshagiri Rao S V, (1985) "Hilbert transform in the interpretation of magnetic anomalies of various components due to thin infinite dyke", PAGEOPH, Vol.123, pp 557-566.

[34] Sundararajan N, Umashankar B, Mohan N L \& Seshagiri Rao S V, (1989) "Direct interpretation of magnetic anomalies due to spherical sources-A Hilbert transform method", Geophysical Transactions, Vol.35, No.3, pp 507-512.

[35] Sundararajan N \& Srinivas Y, (1996) “A modified Hilbert transform and its applications to selfpotential interpretation”, Journal of Applied Geophysics, Vol.36, pp. 137-143.

[36] Sundararajan N, Srinivas Y \& Laxminarayana Rao T, (2000) "Sundararajan Transform - a tool to interpret potential field anomalies”, Exploration Geophysics, Vol. 31, No. 4, pp 622 -628.

\section{AUTHORS}

Dr. M. Bhagwan Das post graduated in Mathematics from the Kakatiya University with gold medal followed M.Phil from University of Hyderabad and Ph.D in Mathematics from Osmania University, Hyderabad, India. He is currently head of the department, Mathematics at Sree Triveni Educational Institutions, Hyderabad. He worked on an inversion of geophysical problems using Neural Networks and Hilbert transformation with Prof. N. Sundararajan. His research is centered on development of new algorithms, Neural Networks, Wavelet Transforms, Fractals and their applications etc.

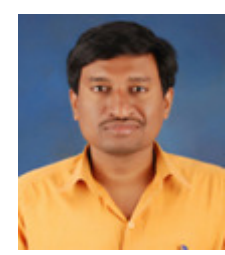

Dr. Narasimman Sundararajan graduated in Mathematics from the University of Madras followed by an M.Sc (Tech) and Ph.D in Geophysics from Osmania University, India. Began a career as a Research Scientist and later switched over to teaching in Osmania University where he became a Professor in 2004. Currently he is in the Department of Earth Sciences, Sultan Qaboos University, Oman. Published more than 90 research papers in the leading International journals besides a book and a couple of Book chapters and supervised several Ph.Ds in Geophysics as well as Mathematics. Brought out a few innovative tools for processing and interpreting of various

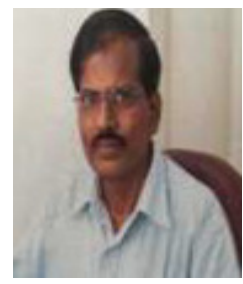
geophysical data besides mathematical concept called "Sundararajan Transform". Implemented several research projects including one on Uranium exploration. Member of XIV Indian Scientific Expedition to Antarctica during 1994-95. Introduced a valid and viable approach to multidimensional Hartley transform in contrast with the definition of Prof R N Bracewell from Stanford University, USA. For his overall significant research contribution, Govt. of India has conferred upon him the National Award for Geosciences in 2007. His research interests are varied and wide including geophysical data processing, mineral and ground water exploration, earth quake hazard assessment studies etc. In 2015, Dr. Sundararajan joined as an Associate Editor of Arabian Journal of Geosciences (Springer) responsible for evaluating submission in the field of theoretical and applied geophysics. 